\title{
ON ANALYTIC PROPERTIES OF DEFORMATION SPACES OF KLEINIAN GROUPS
}

\author{
HIROSHIGE SHIGA
}

\begin{abstract}
Let $G_{0}$ be a non-elementary Kleinian group. We consider the deformation space of $D\left(G_{0}\right)$, the space of quasiconformal deformations of $G_{0}$, and its complex analytic properties. We show some analytic structures of $D\left(G_{0}\right)$ which are improvements of results by Bers, Kra, Maskit and McMullen. In particular, we clarify that the structures for Kleinian groups with non-simply connected components are different from those for Kleinian groups without non-simply connected components.
\end{abstract}

\section{InTRODUCTION AND MAIN RESUltS}

A discrete subgroup $G$ of $\operatorname{PSL}(2, \mathbb{C})$ is called a Kleinian group. It is well known that every Kleinian group $G$ acts on the hyperbolic 3 -space $\mathbb{H}^{3}$ properly discontinuously and it produces a hyperbolic 3 -manifold $\mathbb{H}^{3} / G$. Hence, the deformation of Kleinian groups has its own interest from the viewpoint of the moduli of hyperbolic 3-manifolds as well as of complex analysis. Indeed, a series of works by Bers, Maskit and Kra ([2], [14-[16], 20]) clarified some fundamental properties of quasiconformal deformation spaces of Kleinian groups. In this paper, we consider a global theory of the deformation of Kleinian groups. In particular, we consider complex analytic properties of deformation spaces of Kleinian groups which are improvements of the above works. The main tools in this article are holomorphic motions and Teichmüller theory.

Since every element of $\operatorname{PSL}(2, \mathbb{C})$ is represented by a $2 \times 2$ matrix with complex entries, we may consider a natural complex structure of spaces of Kleinian groups. Before stating our results, we begin with the holomorphic family of Kleinian groups. Let $V$ be a complex manifold with a base point $x_{0}$ and $G_{0}$ be a Kleinian group. We denote by $\operatorname{Hom}\left(G_{0}, \operatorname{PSL}(2, \mathbb{C})\right)$, the set of homomorphisms from $G_{0}$ to $\operatorname{PSL}(2, \mathbb{C})$. We also denote by $\widetilde{\operatorname{Hom}}\left(G_{0}, \operatorname{PSL}(2, \mathbb{C})\right)$ the quotient space of $\operatorname{Hom}\left(G_{0}, \operatorname{PSL}(2, \mathbb{C})\right)$ by conjugation of $\operatorname{PSL}(2, \mathbb{C})$. Both spaces admit the natural complex structures coming from entries of matrices as above.

Suppose that a family of $\left\{\theta_{x}\right\}_{x \in V}$ of $\widetilde{\operatorname{Hom}}\left(G_{0}, \operatorname{PSL}(2, \mathbb{C})\right)$ satisfies the following conditions:

(1) $\theta_{x_{0}}=i d$.

(2) For each $g \in G_{0}$, the map $V \ni z \mapsto \theta_{z}(g) \in \operatorname{PSL}(2, \mathbb{C})$ is holomorphic on $V$.

Received by the editors December 19, 2013 and, in revised form, September 2, 2014.

2010 Mathematics Subject Classification. Primary 32G15; Secondary 30C40, 30F60, 37F30.

Key words and phrases. Kleinian groups, quasiconformal maps, Teichmüller spaces, holomorphic convexity.

The author was partially supported by the Ministry of Education, Science, Sports and Culture, Japan; Grant-in-Aid for Scientific Research (B), 22340028, 2010-2014. 
Then, we call the triple $\mathcal{G}:=\left(G_{0}, V,\left\{\theta_{x}\right\}_{x \in V}\right)$ a holomorphic family of the Kleinian group $G_{0}$ over $V$. If $\theta_{x}$ is isomorphic for every $x \in V$, the family is called a holomorphic family of isomorphisms of $G_{0}$ over $V$. A holomorphic family $\mathcal{G}$ of $G_{0}$ is order preserving if $\theta_{x}(g)$ is of infinite order or the identity for every $g \in G_{0}$ of infinite order.

Note. In the above definition of holomorphic families, condition (2) means that there exist a neighbourhood $U_{z}$ of $z \in V$ and representatives $\rho_{x} \in \operatorname{Hom}\left(G_{0}, \operatorname{PSL}(2, \mathbb{C})\right)$ of $\theta_{x}$ for $x \in U_{z}$ such that the map $U_{z} \ni x \mapsto \rho_{x}(g) \in \operatorname{PSL}(2, \mathbb{C})$ is holomorphic for each $g \in G_{0}$.

Among results on the deformation of Kleinian groups, one important result is a theorem by L. Bers [2, which is on a local theory of the deformation of Kleinian groups. First of all, we recall the theorem of Bers [2] on holomorphic families over the unit disk $\Delta=\{z \in \mathbb{C}|| z \mid<1\}$ (See Corollary 2 in 24 for an infinite version of the theorem.)

Theorem 1.1. Let $G_{0}$ be a non-elementary Kleinian group and $\left\{\theta_{x}\right\}_{x \in \Delta}$ be a holomorphic family of isomorphisms of $G_{0}$ over the unit disk $\Delta$. Suppose that $\theta_{x}\left(G_{0}\right)$ is discrete for any $x \in \Delta$ and $\theta_{x}(g)$ is parabolic whenever $g \in G$ is parabolic. Then, for each $x \in \Delta$ there exists a quasiconformal mapping $w_{x}$ of the Riemann sphere $\hat{\mathbb{C}}$ to itself such that it induces the isomorphism $\theta_{x}$.

We now present the main theorems in our paper; see the following sections for the relevant definitions.

In Theorem 1.1, the assumption that the holomorphic family consists of isomorphisms defined on the unit disk $\Delta$, that is, simply connected, is crucial. If Kleinian groups satisfy a certain condition, we can relax the assumption as follows:

Theorem I. Let $K$ be an AB-removable compact subset of the unit disk $\Delta$ in $\mathbb{C}$. Suppose that $G_{0}$ is a Kleinian group whose components are all simply connected. Then, any order preserving holomorphic family $\mathcal{G}=\left(G_{0}, \Delta_{K},\left\{\theta_{x}\right\}_{x \in \Delta_{K}}\right)$ of $G_{0}$ over $\Delta_{K}:=\Delta \backslash K$ can be extended to a holomorphic family $\widetilde{\mathcal{G}}=\left(G_{0}, \Delta,\left\{\tilde{\theta}_{x}\right\}_{x \in \Delta}\right)$ of $G_{0}$ over $\Delta$.

Immediately, we have the following corollary which is a generalization of the disk convexity of $\mathcal{Q} \mathcal{F}\left(\Gamma_{0}\right)$, the space of quasi-Fuchsian groups of a Fuchsian group $\Gamma_{0}$, by McMullen [23].

Corollary 1.1. Suppose that $G_{0}$ is a Kleinian group whose components are all simply connected. Let $K$ be an AB-removable compact subset of $\Delta$ and $\varphi$ be a holomorphic map from $\Delta_{K} \cup \partial \Delta$ to $\widetilde{\operatorname{Hom}}\left(G_{0}, P S L(2, \mathbb{C})\right)$ so that $\varphi(z)(\gamma)$ is parabolic whenever $\gamma \in G_{0}$ is parabolic. If $\varphi(\partial \Delta) \subset D\left(G_{0}\right)$, then $\varphi$ can be extended to a holomorphic map $\tilde{\varphi}$ on $\Delta$ and $\tilde{\varphi}(\Delta) \subset D\left(G_{0}\right)$.

Remark 1.1. (1) In Theorem \and Corollary 1.1, we do not assume that Kleinian groups are finitely generated.

(2) The statement of the corollary does not hold if we replace $\Delta_{K}$ by an annulus. Namely, for a Fuchsian group $\Gamma_{0}$, we can construct a holomorphic map $\varphi: U \rightarrow \mathcal{Q F}\left(\Gamma_{0}\right)$ from any given annulus $U=\{z \in \Delta|0<r<| z \mid<1\}$ with $r \in(0,1)$ such that $\varphi(\partial U) \subset \mathcal{Q F}\left(\Gamma_{0}\right), \varphi(z)(\gamma)(z \in U)$ is parabolic whenever $\gamma \in \Gamma_{0}$ is parabolic but $\varphi$ cannot be extended to a holomorphic map on the unit disk $\Delta$ (see Remark 4.1). 
In Theorem [1] we cannot drop the assumption that the Kleinian group has only simply connected components. In fact, we may show that Theorem [is not true if the Kleinian group has a non-simply connected component.

Theorem II. Let $G_{0}$ be a finitely generated Kleinian group. Suppose that there exists a non-simply connected component of $G_{0}$. Then, there exists a holomorphic family $\mathcal{G}$ of isomorphisms of $G_{0}$ over the punctured disk $\Delta^{*}:=\{0<|z|<1\}$ such that it cannot be extended to a holomorphic family of $G_{0}$ over $\Delta$.

While Theorem II has its own interest, the theorem also helps us to show the following complex analytic properties of deformation spaces of Kleinian groups.

Theorem III. Let $G_{0}$ be a finitely generated Kleinian group. Then

(1) The Carathéodory pseudo distance on $D\left(G_{0}\right)$ is a distance.

(2) If every component of $G_{0}$ is simply connected, then in $D\left(G_{0}\right)$ the Carathéodory distance is complete. In particular, $D\left(G_{0}\right)$ is $H^{\infty}$-convex.

(3) If there exists a non-simply connected component of $G_{0}$, then $D\left(G_{0}\right)$ is not $H^{\infty}$-convex. In particular, the Carathéodory distance is not complete in $D\left(G_{0}\right)$.

It is well known that in Teichmüller space, the Kobayashi distance and the Teichmüller distance are the same. We show that it also holds for our deformation spaces.

Theorem IV. Let $G_{0}$ be a Kleinian group. Then the Teichmüller distance $d_{T}^{D\left(G_{0}\right)}$ of $D\left(G_{0}\right)$ is equal to the Kobayashi distance $d_{K}^{D\left(G_{0}\right)}$ of $D\left(G_{0}\right)$. In particular, the Kobayashi distance of $D\left(G_{0}\right)$ is complete.

The Kobayashi distance of $D\left(G_{0}\right)$ is complete for every Kleinian group $G_{0}$ while the Carathéodory distance is not complete in $D\left(G_{0}\right)$ if the Kleinian group $G_{0}$ has a non-simply connected component. The following is an immediate consequence.

Corollary 1.2. Let $G_{0}$ be a finitely generated Kleinian group with a non-simply connected component. Then, the Kobayashi distance does not coincide with the Carathéodory distance in $D\left(G_{0}\right)$.

\section{Fundamental nOtions And TERMinologies}

2.1. Holomorphic convexities. We begin with the holomorphic convexity of complex manifolds. Let $M$ be a (connected) complex manifold and $\mathcal{O}(M)$ the space of holomorphic functions on $M$. For a subset $\mathcal{O}$ of $\mathcal{O}(M), M$ is called $\mathcal{O}$ convex or convex for the space $\mathcal{O}$ if for any compact subset $K$ of $M$, the $\mathcal{O}$-convex hull $\hat{K}_{\mathcal{O}}$ of $K$ is compact in $M$, where

$$
\hat{K}_{\mathcal{O}}=\left\{p \in M|| f(p)\left|\leq \max _{z \in K}\right| f(z) \mid \text { for all } f \in \mathcal{O}\right\} .
$$

A complex manifold $M$ is called holomorphically convex if it is $\mathcal{O}(M)$-convex. A domain in $\mathbb{C}^{n}$ is holomorphically convex if and only if it is a domain of holomorphy (cf. [8]). If $\mathcal{O}$ is $H^{\infty}(M)$, the space of bounded holomorphic functions on $M$, and if $M$ is $H^{\infty}(M)$-convex, then we call the manifold $H^{\infty}$-convex. Obviously, if $M$ is $H^{\infty}$-convex, then it is holomorphically convex. It is known that if the 
Carathéodory distance $c_{M}$ of $M$ is complete, then $M$ is $H^{\infty}$-convex. Here, the Carathéodory (pseudo) distance $c_{M}$ of $M$ is defined by

$$
c_{M}(x, y)=\sup _{f \in H_{1}^{\infty}(M)} \rho_{\Delta}(f(x), f(y))
$$

for $x, y \in M$, where $H_{1}^{\infty}(M)$ is the set of holomorphic functions $f$ on $M$ with $\|f\|_{\infty}<1$ and $\rho_{\Delta}$ is the Poincaré distance of the unit disk $\Delta$. In general, the Carathéodory distance is a pseudo distance. Namely, $c_{M}(x, y)=0$ does not necessarily imply $x=y$. The Carathéodory distance is not greater than the Kobayashi distance.

If $M$ is a domain in $\mathbb{C}^{n}$ and it is convex for the space of polynomials in $\mathbb{C}^{n}$, then $M$ is called polynomial convex. It is also easy to see that if $M$ is polynomial convex, then it is $H^{\infty}$-convex.

2.2. Teichmüller spaces. In the following, we shall give a brief discussion on Teichmüller spaces. The reader may refer to [10, 11] or [17] for further details.

Let $X_{0}$ be a Riemann surface of type $(g, n)$ with $2 g-2+n>0$. The Teichmüller space $T\left(X_{0}\right)$ of $X_{0}$ is the set of Teichmüller equivalence classes $[X, f]$ of marked Riemann surfaces $(X, f)$, where a marked Riemann surface is a pair $(X, f)$ of a Riemann surface $X$ and a quasiconformal homeomorphism $f$ from $X_{0}$ onto $X$, and two marked Riemann surfaces $(X, f),\left(X^{\prime}, f^{\prime}\right)$ are Teichmüller equivalent if there exists a conformal mapping $h$ from $X$ to $X^{\prime}$ which is homotopic to $f^{\prime} \circ f^{-1}$.

The Teichmüller space $T\left(X_{0}\right)$ admits a natural complex structure and it is realized as a contractible bounded domain in $\mathbb{C}^{3 g-3+n}$. The Teichmüller space $T\left(X_{0}\right)$ also has a complete distance $d_{T}$ called the Teichmüller distance defined by

$$
d_{T}\left([X, f],\left[X^{\prime}, f^{\prime}\right]\right)=\inf _{g} \log K(g),
$$

where the infimum is taken over all quasiconformal mappings $g$ homotopic to $f^{\prime} \circ f^{-1}$ and $K(g)$ is the maximal dilatation of $g$. It is known that the Teichmüller distance coincides with the Kobayashi distance with respect to the complex structure of $T\left(X_{0}\right)$. Moreover, in the Teichmüller space $T\left(X_{0}\right)$, the Carathéodory pseudo distance $c_{T\left(X_{0}\right)}$ is a distance and it is complete $([3],[26])$.

The Teichmüller distance between $[X, f]$ and $\left[X^{\prime}, f^{\prime}\right]$ is actually achieved by a quasiconformal mapping $f_{X, X^{\prime}}$ from $X$ to $X^{\prime}$ called the Teichmüller mapping, whose Beltrami coefficient is $k|q| / q$, where $k \in[0,1)$ is given by an equation

$$
d_{T}\left([X, f],\left[X^{\prime}, f^{\prime}\right]\right)=\log \frac{1+k}{1-k},
$$

and $q$ is an integrable holomorphic quadratic differential on $X$ with $\int_{X}|q|=1$. Moreover, those $q$ and $k$ are uniquely determined by $\left[X^{\prime}, f^{\prime}\right]$.

Let $\operatorname{Mod}\left(X_{0}\right)$ be the group of homotopy classes $[\omega]$ of quasiconformal self-maps $\omega$ of $X_{0}$, which is called the mapping class group. The mapping class group $\operatorname{Mod}\left(X_{0}\right)$ acts on $T\left(X_{0}\right)$ by

$$
[\omega]([X, f])=\left[X, f \circ \omega^{-1}\right] \quad\left([\omega] \in \operatorname{Mod}\left(X_{0}\right)\right) .
$$

It is easily seen that the action of $\operatorname{Mod}\left(X_{0}\right)$ on $T\left(X_{0}\right)$ is isometric with respect to the Teichmüller distance $d_{T}$. 
Teichmüller disks. Let $q$ be a holomorphic quadratic differential on $X_{0}$ with $\int_{X_{0}}|q|=1$. We consider a holomorphic map from $\Delta$ to the space of Beltrami coefficients by

$$
\Delta \ni t \mapsto t \frac{|q|}{q}
$$

Since the Beltrami coefficient $t|q| / q$ determines a point in $T\left(X_{0}\right)$, the map yields a holomorphic map $\Phi_{q}$ from $\Delta$ to $T\left(X_{0}\right)$. Furthermore, the map $\Phi_{q}$ is isometric with respect to the hyperbolic distance on $\Delta$ and the Teichmüller distance on $T\left(X_{0}\right)$ and the image $\mathbb{D}[q]:=\Phi_{q}(\Delta)$ in $T\left(X_{0}\right)$ is totally geodesic. The image $\mathbb{D}[q]$ is called a Teichmüller disk.

Jenkins-Strebel differentials. Let $q$ be an integrable holomorphic quadratic differential on $X_{0}$. Then a holomorphic germ

$$
X_{0} \ni z \mapsto \int^{z} \sqrt{q} \in \mathbb{C}
$$

gives a flat structure on $X_{0}$ except for zeros of $q$. A horizontal line of the flat structure is called a horizontal trajectory of $q$. If an integrable holomorphic quadratic differential $q$ has a horizontal trajectory which is a simple closed curve on $X_{0}$ and the set of simple closed horizontal trajectories is dense in $X_{0}$, then the holomorphic quadratic differential $q$ is called a Jenkins-Strebel differential. A Jenkins-Strebel differential $q$ is called single if any two closed trajectory of $q$ is homotopic to each other. It is known that for any non-trivial and non-peripheral simple closed curve $\alpha$ in $X_{0}$ there exists a single Jenkins-Strebel differential whose simple closed trajectories are homotopic to $\alpha$.

Let $q$ be a single Jenkins-Strebel differential. Then, the union of closed trajectories makes an annulus $A_{q}$ in $X_{0}$ and the core curve $\alpha_{q}$ of $A_{q}$ is homotopic to a closed trajectory of $q$. We consider the right Dehn twist $\tau_{q} \in \operatorname{Mod}\left(X_{0}\right)$ about $\alpha_{q}$. Then the following theorem is known:

Theorem 2.1 (Marden-Masur [19]). Let q be a single Jenkins-Strebel differential of $X_{0}$ and $A_{q}, \alpha_{q}$ be the same ones as above. Then, the right Dehn twist $\tau_{q} \in \operatorname{Mod}\left(X_{0}\right)$ preserves the Teichmüller disk $\mathbb{D}[q]$ given by $q$. Furthermore, $\Phi_{q}^{-1} \circ \tau_{q} \circ \Phi_{q}: \Delta \rightarrow \Delta$ is a parabolic Möbius transformation on $\Delta$.

2.3. Kleinian groups. For a Kleinian group $G$, the closure of the set of fixed points of elements of $G$ with infinite order is called the limit set of $G$ and it is denoted by $\Lambda(G)$. If the limit set $\Lambda(G)$ contains infinitely many points, then the Kleinian group $G$ is called non-elementary. Throughout this paper, we assume that every Kleinian group is non-elementary.

The complement of the limit set $\Lambda(G)$ in $\hat{\mathbb{C}}$ is called the region of discontinuity of $G$ and it is denoted by $\Omega(G)$. By the definition, $\Omega(G)$ is an open set in $\hat{\mathbb{C}}$ but it may be empty. A connected component of $\Omega(G)$ is called a component of $G$. It is known that $\Omega(G)$ is the maximal open set where the action of $G$ is properly discontinuous. Hence, the quotient space $\Omega(G) / G$ consists of Riemann surfaces (or orbifolds) if $\Omega(G)$ is not empty. Furthermore, we have the following:

Theorem 2.2 (Ahlfors' finiteness theorem; cf. [22]). Suppose that $G$ is a finitely generated Kleinian group with non-empty region of discontinuity. Then $\Omega(G) / G$ is a finite union of hyperbolic Riemann surfaces of finite type. 
A Riemann surface is called of finite type if it is obtained by removing at most finitely many points from a compact Riemann surface.

Every element $g$ of $\operatorname{PSL}(2, \mathbb{C})$ can be extended to the upper half space $\mathbb{H}^{3}=$ $\left\{(x, y, t) \mid x, y \in \mathbb{R}, t \in \mathbb{R}_{+}\right\}$as a hyperbolic isometry. Hence, a Kleinian group $G$ acts on the hyperbolic 3 -space $\mathbb{H}^{3}$. Moreover, the action is properly discontinuous and we obtain a hyperbolic 3-manifold (or orbifold) $M(G)=\mathbb{H}^{3} / G$ and the boundary $\partial M(G)$ of $M(G)$ is $\Omega(G) / G$. While there are many aspects and results on hyperbolic 3-manifolds and Kleinian groups, we present the following theorem which is used later in this paper.

Theorem 2.3 (The loop theorem; cf. [20]). Let $M$ be a 3-manifold with boundary and $B$ be a connected component of the boundary of $M$. Suppose that there exists a non-trivial loop $\alpha$ in $B$ which is trivial in $M$. Then there exists a non-trivial simple loop $\beta$ in $B$ which is trivial in $M$.

2.4. The deformation space of Kleinian groups. Let $G_{0}$ be a finitely generated non-elementary Kleinian group. The deformation space $D\left(G_{0}\right)$ of $G_{0}$ is the space of $\theta \in \widetilde{\operatorname{Hom}}\left(G_{0}, \operatorname{PSL}(2, \mathbb{C})\right)$ given by normalized quasiconformal mappings on $\hat{\mathbb{C}}$. Namely, $\theta \in \widetilde{\operatorname{Hom}}\left(G_{0}, \operatorname{PSL}(2, \mathbb{C})\right)$ belongs to $D\left(G_{0}\right)$ if and only if there exists a quasiconformal self-map $w$ of $\hat{\mathbb{C}}$ fixing 0,1 and $\infty$ such that $\theta$ is represented by an isomorphism of $G_{0}$ to $\operatorname{PSL}(2, \mathbb{C})$ given by

$$
G_{0} \ni g \mapsto w g w^{-1} \in \operatorname{PSL}(2, \mathbb{C}) .
$$

In [16] and [21, it is shown that $D\left(G_{0}\right)$ is a complex manifold and it is holomorphically convex. Recently, Earle and Marden [5] discuss the deformation spaces with holomorphic families of Riemann surfaces.

On the space $D\left(G_{0}\right)$, we may define the Teichmüller distance $d_{T}^{D\left(G_{0}\right)}$ :

$$
d_{T}^{D\left(G_{0}\right)}\left(\theta_{1}, \theta_{2}\right)=\inf _{w_{1}, w_{2}} \log K\left(w_{2} \circ w_{1}^{-1}\right) \quad\left(\theta_{1}, \theta_{2} \in D\left(G_{0}\right)\right),
$$

where $w_{i}(i=1,2)$ are taken over all quasiconformal self-maps of $\hat{\mathbb{C}}$ giving $\theta_{i}$. A standard argument of quasiconformal mappings shows that the Teichmüller distance $d_{T}^{D\left(G_{0}\right)}$ is complete in $D\left(G_{0}\right)$.

Let $X_{0}$ be a hyperbolic Riemann surface of finite type and $\Gamma_{0}$ be a Fuchsian group acting on $\mathbb{H}$ with $X_{0}=\mathbb{H} / \Gamma_{0}$. We denote by $\mathcal{Q F}\left(\Gamma_{0}\right)$ the set of all conjugacy classes of isomorphisms of $\Gamma_{0}$ to $\operatorname{PSL}(2, \mathbb{C})$ given by a normalized quasiconformal mapping of $\hat{\mathbb{C}}$. The space $\mathcal{Q F}\left(\Gamma_{0}\right)$ is called the space of quasi-Fuchsian groups for $\Gamma_{0}$. It is actually the deformation space $D\left(\Gamma_{0}\right)$ and it is regarded as a subset of $\widetilde{\operatorname{Hom}}\left(\Gamma_{0}, \operatorname{PSL}(2, \mathbb{C})\right)$, the space of representations of the Fuchsian group $\Gamma_{0}$ to $\operatorname{PSL}(2, \mathbb{C})$.

The simultaneous uniformization theorem by Bers ([1] $)$ implies that $\mathcal{Q F}\left(\Gamma_{0}\right)$ can be identified with $T\left(X_{0}\right) \times T\left(\overline{X_{0}}\right)$, the product space of the Teichmüller spaces, where $\overline{X_{0}}$ is the complex conjugate of the Riemann surface $X_{0}$. Moreover, it has a natural complex structure. Under the complex structure, McMullen ([23]) shows the following disk convexity:

Theorem 2.4. Let $\varphi$ be a holomorphic map from $\bar{\Delta}$ to $\widetilde{\operatorname{Hom}}\left(\Gamma_{0}, P S L(2, \mathbb{C})\right)$ so that $\varphi(z)(\gamma)$ is parabolic for every parabolic element $\gamma \in \Gamma_{0}$. Suppose that $\varphi(\partial \Delta) \subset$ $\mathcal{Q F}\left(\Gamma_{0}\right)$. Then, $\varphi(\Delta) \subset \mathcal{Q F}\left(\Gamma_{0}\right)$. 


\section{Holomorphic motions}

In this section, we shall give some fundamental facts on holomorphic motions used in this paper.

Let $E$ be a subset of $\hat{\mathbb{C}}$ containing at least three points and $V$ be a connected complex manifold with a base point $x_{0}$. A map $\phi: V \times E \rightarrow \hat{\mathbb{C}}$ is called a holomorphic motion of $E$ over $V$ if it satisfies the following conditions:

(1) $\phi\left(x_{0}, \cdot\right)$ is the identity on $E$;

(2) for each $x \in V, \phi(x, \cdot)$ is injective on $E$;

(3) for each $z \in E, \phi(\cdot, z)$ is a holomorphic function on $V$.

A holomorphic motion $\phi: V \times E \rightarrow \hat{\mathbb{C}}$ is called normalized if $E$ contains 0,1 and $\infty$ and $\phi(x, \cdot)$ fixes 0,1 and $\infty$ for any $x \in V$. From now on, we assume that a holomorphic motion is always normalized since every holomorphic motion is conjugate to a normalized one by Möbius transformations.

A striking theorem on holomorphic motions is the following theorem by Slodkowski [27]:

Theorem 3.1 (Slodkowski). Let $E$ be a subset of $\hat{\mathbb{C}}$ containing at least three points. Then every holomorphic motion of $E$ over the unit disk $\Delta$ in $\mathbb{C}$ can be extended to a holomorphic motion of $\hat{\mathbb{C}}$ over $\Delta$.

Furthermore, Earle, Kra and Krushkal' (4]) showed that Theorem 3.1 is still true for holomorphic motions which are Möbius group equivariant as follows;

Theorem 3.2. Let $G$ be a group of Möbius transformations and $E \subset \hat{\mathbb{C}}$ a closed set invariant for $G$. Suppose that a holomorphic motion $\phi: \Delta \times E \rightarrow \hat{\mathbb{C}}$ is $G$ equivariant on $E$, that is, there exists an isomorphism $\rho_{\lambda}: G \rightarrow \operatorname{PSL}(2, \mathbb{C})$ for each $\lambda \in \Delta$ such that

$$
\phi(\lambda, g(z))=\rho_{\lambda}(g)(\phi(\lambda, z)) \quad(z \in E) .
$$

Then $\phi$ extends a holomorphic motion $\tilde{\phi}: \Delta \times \hat{\mathbb{C}}$ of $\hat{\mathbb{C}}$ over $\Delta$ which is G-equivariant on $\hat{\mathbb{C}}$.

On the other hand, it is known that then there exist a simply connected complex manifold $V$ with $\operatorname{dim} V \geq 2$ and a holomorphic motion of a finite set over $V$ such that it cannot be extended to a holomorphic motion of $\hat{\mathbb{C}}$ over $V$ which is a consequence of a theorem by Hubbard ([9]). See also [12 for an easier example.

We consider conditions for holomorphic motions of $E$ over $V$ to be extended to holomorphic motions of $\hat{\mathbb{C}}$ over $V$ when $\operatorname{dim} V=1$ and $V$ is not simply connected. In particular, we consider $V=\Delta_{K}:=\Delta \backslash K$ for an $A B$-removable compact subset $K$ of $\Delta$ as in our main theorems. We say that a compact subset $K$ of $\Delta$ is $A B$ removable if any bounded holomorphic function in $\Delta_{K}$ is extended to a holomorphic function in $\Delta$. Obviously, $K$ is nowhere dense and $\Delta_{K}$ is connected.

The concept of "monodromy" of a holomorphic motion was studied in [1]; see Section 2.4 of that paper. For the reader's convenience, we include here a brief discussion.

We assume that $E$ is a finite set and it contains $n(>3)$ points including 0,1 and $\infty$. Let $\phi: V \times E \rightarrow \hat{\mathbb{C}}$ be a holomorphic motion. Consider a holomorphic universal covering $\pi: \widetilde{V} \rightarrow V$ with the cover transformation group $G_{V}$ and take a point $\widetilde{x}_{0} \in \widetilde{V}$ so that $\pi\left(\widetilde{x}_{0}\right)=x_{0}$. Then $\Phi=\pi^{*}(\phi): \widetilde{V} \times E \rightarrow \hat{\mathbb{C}}$ is a holomorphic 
motion of $E$ over $\widetilde{V}$ with $\widetilde{x}_{0}$ as the base point. Then, there exists a base point preserving continuous map $f: \widetilde{V} \rightarrow M(\mathbb{C})$ such that

$$
\Phi(x, z)=w^{f(x)}(z) \text { for each } x \in \widetilde{V} \text { and each } z \in E,
$$

where $M(\mathbb{C})$ is the space of Beltrami coefficients on $\mathbb{C}$,

$$
w^{f \circ g\left(\widetilde{x}_{0}\right)}(z)=\Phi\left(g\left(\widetilde{x}_{0}\right), z\right)=\phi\left(\pi \circ g\left(\widetilde{x}_{0}\right), z\right)=\phi\left(x_{0}, z\right)=z .
$$

Therefore, $w^{f \circ g\left(\widetilde{x}_{0}\right)}$ keeps every point of $E$ fixed.

It is not hard to see that the homotopy class for $w^{f \circ g\left(\widetilde{x}_{0}\right)}$ relative to $E$ is well defined, that is, it does not depend on the choice of continuous mappings $f$. The map $w^{f \circ g\left(\widetilde{x}_{0}\right)}$ is a quasiconformal self-map of the Riemann surface $X_{E}:=\hat{\mathbb{C}} \backslash E$. Therefore, it represents a mapping class of $X_{E}$, and by Lemma 2.12 in [1, we have a homomorphism $\sigma_{\phi}: \pi_{1}\left(V, x_{0}\right) \rightarrow \operatorname{Mod}(0, n)$ given by

$$
\sigma_{\phi}(c)=\left[w^{f \circ g_{c}\left(\widetilde{x}_{0}\right)}\right]
$$

where $\operatorname{Mod}(0, n)$ is the mapping class group of the $n$-times punctured sphere, $g_{c} \in$ $G_{V}$ is an element corresponding to $c \in \pi_{1}\left(V, x_{0}\right)$, and $[w]$ denotes the mapping class of $X_{E}$ for $w$. We call the homomorphism $\sigma_{\phi}$ the monodromy of the holomorphic motion $\phi$ of the finite set $E$. The monodromy is called trivial if it maps every element of $\pi_{1}\left(V, x_{0}\right)$ to the identity of $\operatorname{Mod}(0, n)$.

If $\phi$ extends to a holomorphic motion of $\hat{\mathbb{C}}$, then it represents a holomorphic family of quasiconformal mappings with the parameter space $V$. This gives us the following (cf. [1])

Proposition 3.1. Let $\phi: V \times E \rightarrow \hat{\mathbb{C}}$ be a holomorphic motion of a finite set $E$. If $\phi$ can be extended to a holomorphic motion of $\hat{\mathbb{C}}$ over $V$, then the monodromy $\sigma_{\phi}$ is trivial.

If $V=\Delta_{K}:=\Delta \backslash K$ for an $A B$-removable compact subset $K$ of $\Delta$, we can say more $([1])$.

Proposition 3.2. Let $\phi: \Delta_{K} \times E \rightarrow \hat{\mathbb{C}}$ be a holomorphic motion of $E$ over $\Delta_{K}$. Then, the holomorphic motion $\phi$ can be extended to a holomorphic motion of $\hat{\mathbb{C}}$ if and only if the monodromy of $\left.\phi\right|_{E^{\prime}}$ is trivial for any finite subset $E^{\prime}$ of $E$, where $\left.\phi\right|_{E^{\prime}}$ is a holomorphic motion of $E^{\prime}$ over $\Delta_{K}$ defined by $\left.\phi\right|_{E^{\prime}}(x, z)=\phi(x, z)$ for $(x, z) \in \Delta_{K} \times E^{\prime}$.

By using the above proposition, we can show the following:

Proposition 3.3 ([1]). Let $K$ be an AB-removable compact subset of $\Delta$ and $E$ be a closed set containing 0,1 and $\infty$.

(1) If every connected component of the complement of $E$ is either simply connected or conformally equivalent to the punctured disk, then every holomorphic motion of $E$ over $\Delta_{K}$ can be extended to a holomorphic motion of $\hat{\mathbb{C}}$ over $\Delta_{K}$

(2) Suppose that $E$ consists of more than five points. If some component of the complement of $E$ is neither simply connected nor conformally equivalent to the punctured disk, then there exists a holomorphic motion of $E$ over $\Delta_{K}$ such that it cannot be extended to a holomorphic motion of $\hat{\mathbb{C}}$ over $\Delta_{K}$. 
Proof. While the proof is given in [1, we will show a sketch of the proof of (2) since it shows the difficulty of the proof of Theorem $\amalg$

Let $K$ be an $A B$-removable compact subset of $\Delta$. We may assume that $K$ contains the origin. Let $E$ be a closed set satisfying the conditions of (2) and $\Omega$ a connected component of $E^{c}$ which is neither simply connected not conformally equivalent to the punctured disk. Since $E$ contains at least five points, there exists a simply connected domain $D$ such that $\partial D \subset \Omega, D$ contains at least two points of $E$, say $z_{1}, z_{2}$, and $D^{c} \cap E$ contains at least three points. We may assume that 0,1 and $\infty$ are not in $D$. We take $z_{0}$ in $D \backslash E$.

Let $h: \Delta \rightarrow D$ be a Riemann map with $h(0)=z_{0}$. Then, there exists a positive number $r<1$ such that $h(\{r<|x|<1\}) \cap E=\emptyset$. We construct a holomorphic motion $\phi_{0}$ of $E$ over $\Delta^{*}$ as follows for $x \in \Delta^{*}$ :

$$
\phi_{0}(x, z)= \begin{cases}z, & z \in E \backslash D, \\ h\left(x h^{-1}(z) / r\right), & z \in E \cap D .\end{cases}
$$

Then, we can see that the monodromy of $\phi_{0}$ about the origin is not trivial. Hence, it follows from Proposition 3.2 that $\phi_{0}$ cannot be extended to a holomorphic motion of $\hat{\mathbb{C}}$ over $\Delta_{K}$.

\section{Proof of Theorem I}

Let $\mathcal{G}=\left(G_{0}, \Delta_{K},\left\{\theta_{x}\right\}_{x \in \Delta_{K}}\right)$ be an order preserving holomorphic family of $G_{0}$ over $\Delta_{K}$. First, we show that the family is a holomorphic family of isomorphisms.

Indeed, if there exists $x \in \Delta_{K}$ such that $\theta_{x}$ is not an isomorphism, then the kernel $\Gamma_{0}$ of $\theta_{x}$ is a non-trivial normal subgroup of $G_{0}$. Since $G_{0}$ is non-elementary, $\Gamma_{0}$ contains a loxodromic element $g$. Noting that the trace function $f(z):=\operatorname{Tr}^{2} \theta_{z}(g)$ $\left(z \in \Delta_{K}\right)$ is invariant for the conjugation of $\operatorname{PSL}(2, \mathbb{C})$ and $\theta_{x_{0}}=i d$, we see that $f$ is holomorphic and $f(x)=4$ but $f\left(x_{0}\right) \neq 4$. Thus, $f$ is a non-constant holomorphic function on $\Delta_{K}$. Hence it is an open map and we see that there exists $y \in \Delta_{K}$ near $x$ such that $\theta_{y}(g)$ is an elliptic element of finite order. It is a contradiction. Thus, the family is a holomorphic family of isomorphisms.

By the same argument, we see that every $\theta_{x}\left(x \in \Delta_{K}\right)$ is type preserving, that is, $\theta_{x}(g)$ is parabolic if and only if $g \in G_{0}$ is parabolic. Indeed, if $\theta_{x}(g)$ is parabolic for some loxodromic element $g \in G_{0}$, then we verify that there exists a point $y \in \Delta_{K}$ such that $\theta_{y}(g)$ is an elliptic element of finite order. It is absurd because $\theta_{y}$ is order preserving (and isomorphic).

Therefore, from the Sullivan-Riley theorem (25], 28]) we see that every $G_{x}:=$ $\theta_{x}\left(G_{0}\right)$ is discrete $\left(x \in \Delta_{K}\right)$. By Theorem 1.1 we see that the family $\left\{G_{x}\right\}_{x \in \Delta_{K}}$ has the following property: for a simply connected neighbourhood $U$ of a point $x$ in $\Delta_{K}$, there exists a quasiconformal mapping $w_{y}$ for each $y \in U$ such that $\theta_{y} \circ \theta_{x}^{-1}$ is conjugate to the isomorphism:

$$
G_{0} \ni g \mapsto w_{y} \circ g \circ w_{y}^{-1} \in \operatorname{PSL}(2, \mathbb{C}) .
$$

Consider two loxodromic elements $\gamma_{1}, \gamma_{2} \in G_{0}$. We may assume that the attracting fixed point of $\gamma_{1}$ is 0 , the repelling fixed point of $\gamma_{1}$ is 1 and the attracting fixed point of $\gamma_{2}$ is $\infty$. For $x \in \Delta_{K}$, we take a simply connected domain $U$ in $\Delta_{K}$ containing $x$ and the base point $x_{0}$. Since $U$ is conformally equivalent to $\Delta$, it follows from Theorem 1.1 that there exists a normalized quasiconformal map $w_{x}^{U}$ for each $x \in U$ such that it gives the conjugacy class of $\theta_{x}$. The normalized quasiconformal 
map $w_{x}^{U}$ does not depend only on $x \in \Delta_{K}$. However, we may show that $\left.w_{x}^{U}\right|_{\Lambda\left(G_{0}\right)}$ depends only on $x$.

Lemma 4.1. The map $\left.w_{x}^{U}\right|_{\Lambda\left(G_{0}\right)}$ does not depend on the choice of $U$. It depends only on $x \in \Delta_{K}$.

Proof. We consider another simply connected domain $V$ in $\Delta_{K}$ containing $x$ and $x_{0}$. Then, we may also take a normalized quasiconformal map $w_{x}^{V}$ giving the conjugacy class of $\theta_{x}$. Hence, there exists $A \in \operatorname{PSL}(2, \mathbb{C})$ such that $w_{x}^{U}$ and $A \circ w_{x}^{V}$ define the same isomorphism of $G_{0}$. In particular, we have

$$
w_{x}^{U} \circ \gamma_{i} \circ\left(w_{x}^{U}\right)^{-1}=A \circ w_{x}^{V} \circ \gamma_{i} \circ\left(w_{x}^{V}\right)^{-1} \circ A^{-1} \quad(i=1,2) .
$$

Because of the normalization of fixed points of $\gamma_{1}$ and $\gamma_{2}$ as above, we see that $A$ must fix the three points, 0,1 and $\infty$ and $A$ is the identity. So, $w_{x}^{V}$ defines the same isomorphism $\rho_{x}$ of $G_{0}$ as $w_{x}^{U}$. Therefore, we have

$$
\left.w_{x}^{U}\right|_{\Lambda\left(G_{0}\right)}=\left.w_{x}^{V}\right|_{\Lambda\left(G_{0}\right)} .
$$

Thus, $\phi(x, z):=w_{x}^{U}(z)$ is independent of the choice of $U$ and it is well defined on $\Delta_{K} \times \Lambda\left(G_{0}\right)$. Obviously, $\phi(x, \cdot)=w_{x}^{U}(\cdot)$ is injective on $\Lambda\left(G_{0}\right)$ and $\phi\left(x_{0}, \cdot\right)=i d$.

Let $L_{0}$ be the set of loxodromic fixed points of $G_{0}$. For a loxodromic Möbius transformation $g$, we denote by $\alpha(g)$ the attractive fixed point of $g$. Since the quasiconformal map $w_{x}^{U}$ sends the attractive fixed point of $\gamma \in G_{0}$ to that of $\gamma_{x}:=w_{x}^{U} \circ \gamma \circ\left(w_{x}^{U}\right)^{-1}$, we verify that for each loxodromic transformation $\gamma \in G_{0}$, $\phi(x, \alpha(\gamma))=w_{x}^{U}(\alpha(\gamma))$ is holomorphic on $\Delta_{K}$ and $\phi(\cdot, \cdot)$ is a holomorphic motion of $L_{0}$ over $\Delta_{K}$.

It is known that $\overline{L_{0}}=\Lambda\left(G_{0}\right)$. Thus, the holomorphic motion $\phi$ of $L_{0}$ can be extended to a holomorphic motion of $\Lambda\left(G_{0}\right)$ over $\Delta_{K}$ (cf. [18]). We use the same letter $\phi$ for the extended holomorphic motion of $\Lambda\left(G_{0}\right)$. We also see that the holomorphic motion $\phi$ is $G_{0}$-equivariant, namely

$$
\phi(x, g(z))=\rho_{x}(g)(\phi(x, z))
$$

holds for every $g \in G_{0}$ and for every $z \in \Lambda\left(G_{0}\right)$. Indeed, if $z=\alpha(\gamma) \in L_{0}$ for some $\gamma \in G_{0}$, then $g(z)=\alpha\left(g \gamma g^{-1}\right)$ for $g \in G_{0}$. Thus, we obtain

$$
\phi(x, g(z))=\alpha\left(\rho_{x}\left(g \gamma g^{-1}\right)\right)=\rho_{x}(g)\left(\alpha\left(\rho_{x}(\gamma)\right)\right)=\rho_{x}(g)(\phi(x, z)),
$$

for $z \in L_{0}$. Since $L_{0}$ is dense in $\Lambda\left(G_{0}\right)$, the holomorphic motion $\phi$ is $G_{0}$-equivariant on $\Lambda\left(G_{0}\right)$ because of the continuity of $\phi$. Thus, it follows from Proposition 3.3 (1) and Theorem 2 in 24 that $\phi$ can be extended to a holomorphic motion $\tilde{\phi}$ of $\hat{\mathbb{C}}$ over $\Delta$. We show that $\tilde{\phi}$ is still a $G_{0}$-equivariant holomorphic motion of $\Lambda\left(G_{0}\right)$ over $\Delta$.

Let $x$ be a point in $K$. It suffices to show that $\tilde{\phi}(x, \cdot)$ is $G_{0}$-equivariant on $\Lambda\left(G_{0}\right)$. Since an $A B$-removable set is nowhere dense, we may take a sequence $\left\{x_{n}\right\}_{n=1}^{\infty} \subset \Delta_{K}$ converging to $x$. Taking a subsequence if necessary, we see that there exist normalized quasiconformal maps $w_{n}$ and $w$ such that

(1) $w_{n}$ and $w_{n}^{-1}$ uniformly converges to $w$ and $w^{-1}$ as $n \rightarrow \infty$ on $\hat{\mathbb{C}}$, respectively;

(2) $\tilde{\phi}\left(x_{n}, z\right)=w_{n}(z)$ and $\tilde{\phi}(x, z)=w(z)$.

From (2), we have

$$
w_{n}(g(z))=\tilde{\phi}\left(x_{n}, g(z)\right)=\rho_{x_{n}}(g)\left(\tilde{\phi}\left(x_{n}, z\right)\right)=\rho_{w_{n}}(g)\left(w_{n}(z)\right),
$$


for every $z \in \Lambda\left(G_{0}\right)$. Then, we see that $\rho_{w_{n}}(g)=w_{n} \circ g \circ w_{n}^{-1}$ converges to a homeomorphism on $\Lambda\left(G_{0}\right)$ by (1). Since $\rho_{w_{n}}(g) \in \operatorname{PSL}(2, \mathbb{C})$ and $\Lambda\left(G_{0}\right)$ contains more than three points, the limit $\rho(g)=w \circ g \circ w^{-1}$ also belongs to $\operatorname{PSL}(2, \mathbb{C})$ (cf. 17] $\S 2.2-2.3)$. Obviously, $\rho$ is an isomorphism of $G_{0}$ and we have

$$
\tilde{\phi}(x, g(z))=\rho(g)(\tilde{\phi}(x, z)),
$$

for every $g \in G_{0}$ and $z \in \Lambda\left(G_{0}\right)$. Because of (4.1), the map $\rho(g)$ does not depend on $\left\{x_{n}\right\}$. Thus, $\tilde{\phi}(x, \cdot)$ is $G_{0}$-equivariant on $\Lambda\left(G_{0}\right)$ for $x \in K$.

Since $\tilde{\phi}$ is a $G_{0}$-equivariant holomorphic motion of $\Lambda\left(G_{0}\right)$ over $\Delta$, we obtain a $G_{0}$-equivariant holomorphic motion of $\hat{\mathbb{C}}$ over $\Delta$ from Theorem 3.2 and the proof is completed.

Remark 4.1. It is known that Teichmüller space $T(X)$ of a compact Riemann surface $X$ of genus $g>1$ is regarded as a bounded domain containing the origin in $\mathbb{C}^{3 g-3}$. Thus, we may take an annulus $A \subset \Delta \hookrightarrow T(X) \subset \mathbb{C}^{3 g-3}$. Since $T(X)$ is a subset of $\mathcal{Q} \mathcal{F}(\Gamma)$ for a Fuchsian group $\Gamma$ which represents $X$, it is easy to see that there exists a holomorphic map $\varphi: A \cup \partial A \rightarrow T(X) \subset \mathcal{Q F}(\Gamma)$ such that it cannot be extended to a holomorphic map from $\Delta$ to $\mathcal{Q} \mathcal{F}(\Gamma)$. For example, for $A=\{1<|z|<2\}$, we take $\epsilon>0$ so small that $\varphi(z)=\left(\epsilon z^{-1}, 0, \ldots, 0\right) \in \mathbb{C}^{3 g-3}$ belongs to $T(X)$ for any $z \in A \cup \partial A$. Every $\varphi(z) \in T(X)$ induces a type preserving isomorphism. However, since $T(X)$ is a bounded domain, the map $\varphi$ cannot be extended to a holomorphic map on $\Delta$.

\section{Proof of Theorem II}

Let $\Omega$ be a non-simply connected component of $\Omega\left(G_{0}\right)$ of a finitely generated Kleinian group $G_{0}$. As in the argument in the proof of Proposition 3.3, we may construct a holomorphic motion of $\Lambda\left(G_{0}\right)$ over $\Delta^{*}$ which cannot be extended to a holomorphic motion of $\hat{\mathbb{C}}$. However, it is not $G_{0}$-equivariant at all. The difficulty is to make a $G_{0}$-equivariant holomorphic motion.

For the universal covering $\pi: \Delta \rightarrow \Omega$, we consider

$$
\Gamma_{\Omega}:=\left\{\gamma \in \operatorname{PSL}(2, \mathbb{C}) \mid \pi \circ \gamma=g \circ \pi, \text { for some } g \in G_{0}\right\} .
$$

The group $\Gamma_{\Omega}$ is called the Fuchsian equivalent of $G_{0}$ on $\Omega$ and it is seen that

$$
\Delta / \Gamma_{\Omega}=\Omega / G_{0}^{\Omega}=: X_{\Omega},
$$

where $G_{0}^{\Omega}$ is the set of $g \in G_{0}$ which fix $\Omega$. Since $G_{0}$ is of finitely generated, $X_{\Omega}$ is a Riemann surface of finite type (Theorem 2.2). The main idea of the proof is to construct a holomorphic family of the Kleinian group $G_{0}$ parametrized by $\Delta$ with invariance under the action of some parabolic cyclic group. In fact, the parabolic cyclic group is determined by a Dehn twist on $X_{\Omega}$.

Since $\pi$ is the universal covering map of $\Omega$, for a non-trivial simple closed geodesic $c$ in $\Omega$ we obtain an element $g_{c} \in \Gamma_{\Omega} \backslash\{i d\}$ which represents $c$. The transformation $g_{c} \in \Gamma_{\Omega}$ defines a non-trivial closed curve $\alpha_{c}$ in $X_{\Omega}$. On the other hand, the curve $c$ is not represented by any non-trivial element in $G_{0}$ since it is a closed curve in $\Omega$. Hence, the closed curve $\alpha_{c}$ in $X_{\Omega}$ is trivial in the hyperbolic 3-manifold $M:=\left(\mathbb{H}^{3} \cup \Omega\left(G_{0}\right)\right) / G_{0}$. Thus, from Theorem 2.3 we have a non-trivial simple closed curve $\beta$ in $X_{\Omega}$ which is trivial in $M$. 
We denote by $\tilde{\beta}$ a connected component of the lift of $\beta$ in $\Omega$. Since $\beta$ is trivial in $M$, there exists an annular neighbourhood $A_{\tilde{\beta}}$ of $\tilde{\beta}$ such that the canonical projection $\pi_{\Omega}: \Omega \rightarrow X_{\Omega}$ is injective on $A_{\tilde{\beta}}$.

Now, we consider the right Dehn twist $\tau_{\beta}$ about $\beta$. We may assume that $\tau_{\beta}$ is the identity on $X_{\Omega} \backslash \pi_{\Omega}\left(A_{\tilde{\beta}}\right)$. Therefore, we can lift this to a self-map $\tau_{\tilde{\beta}}$ of $\Omega$. Actually, $\tau_{\tilde{\beta}}$ is the product of the right Dehn twists about $\pi_{\Omega}^{-1}(\beta)\left(=\bigcup_{g \in G_{0}} g(\tilde{\beta})\right)$. Moreover, we see that

for any $z \in \Omega$ and any $g \in G_{0}^{\Omega}$.

$$
g \circ \tau_{\tilde{\beta}}(z)=\tau_{\tilde{\beta}} \circ g(z)
$$

Let $q_{\beta}$ be a single Jenkins-Strebel differential on $X_{\Omega}$ whose closed trajectories are homotopic to $\beta$. We denote by $\Phi_{\beta}: \Delta \rightarrow \mathbb{D}\left[q_{\beta}\right]$ the holomorphic map induced by the map

$$
\Delta \ni t \mapsto t \frac{\left|q_{\beta}\right|}{q_{\beta}}
$$

It follows from Theorem 2.1 that the Teichmüller disk $\mathbb{D}\left[q_{\beta}\right]$ in $T\left(X_{\Omega}\right)$ given by $q_{\beta}$ is preserved by the action of the Dehn twist $\tau_{\beta}$ about $\beta$ and a parabolic Möbius transformation $\gamma$ on $\Delta\left(\simeq \mathbb{D}\left[q_{\beta}\right]\right)$ gives the action of $\tau_{\beta}$. More precisely, for every $z \in \Delta$

$$
\Phi_{\beta}(\gamma(z))=\left[\tau_{\beta}\right]\left(\Phi_{\beta}(z)\right)
$$

holds, where $[\omega]$ means the mapping class of a quasiconformal self-map $\omega$ of $X_{\Omega}$.

Let $f_{\beta}: X_{\Omega} \rightarrow X_{\Omega}$ be a quasiconformal map by a Beltrami coefficient $\gamma(0)\left|q_{\beta}\right| / q_{\beta}$. Since the map represents the right Dehn twist about $\beta$, it is lifted to a quasiconformal map $\tilde{f}_{\beta}: \Omega \rightarrow \Omega$ with

for every $g \in G_{0}^{\Omega}$.

$$
\tilde{f}_{\beta} \circ g=g \circ \tilde{f}_{\beta}
$$

The quasiconformal map $\tilde{f}_{\beta}$ on $\Omega$ is extended to a quasiconformal map on $\Omega\left(G_{0}\right)$ to itself defined by $g \circ \tilde{f}_{\beta} \circ g^{-1}$ on $g(\Omega)$ for $g \in G_{0}$ and the identity on $\Omega\left(G_{0}\right) \backslash$ $\bigcup_{g \in G_{0}} g(\Omega)$. We use the same letter $\tilde{f}_{\beta}$ for the extended map. Then, we have $\tilde{f}_{\beta} \circ g=g \circ \tilde{f}_{\beta}$ for any $g \in G_{0}$ on $\Omega\left(G_{0}\right)$. From Maskit's self-map theorem ([21]), we conclude that $\tilde{f}_{\beta}$ is extended to a quasiconformal map on $\hat{\mathbb{C}}$ which commutes with any $g \in G_{0}$. We also use the same letter $\tilde{f}_{\beta}$ for the extended map and denote by $\mu$ the Beltrami coefficient of $\tilde{f}_{\beta}$. Obviously, the extended map $\tilde{f}_{\beta}$ also satisfies

$$
\tilde{f}_{\beta} \circ g=g \circ \tilde{f}_{\beta}
$$

for every $g \in G_{0}$. Therefore, the Beltrami coefficient $\mu$ is $G_{0^{-}}$compatible. Namely, the Beltrami coefficient $\mu$ satisfies

$$
\mu(g(z)) \overline{g^{\prime}(z)} g^{\prime}(z)^{-1}=\mu(z) \quad \text { a.e. }
$$

for every $g \in G_{0}$. Therefore, a holomorphic map

$$
\Delta \ni t \mapsto t \mu
$$

defines a holomorphic family of Beltrami coefficients which are $G_{0}$-compatible.

From this family, we construct a holomorphic family of normalized quasiconformal mappings $w_{t}$ on $\hat{\mathbb{C}}(t \in \Delta)$. The holomorphic family $\left\{w_{t}\right\}_{t \in \Delta}$ induces a holomorphic family $\left\{\theta_{t}\right\}_{t \in \Delta}$ of isomorphisms of $G_{0}$ over $\Delta$ defined by

$$
\theta_{t}(g)=w_{t} g w_{t}^{-1} \quad\left(g \in G_{0}\right) \text {. }
$$


We may take $w_{0}=i d$ and $w_{\gamma(0)}=\tilde{f}_{\beta}$. Then, we have $\theta_{0}=\theta_{\gamma(0)}$ because of (5.2). By the same reason, we conclude that

$$
\theta_{t}=\theta_{\gamma(t)}
$$

for every $t \in \Delta$. Therefore, the holomorphic family $\mathcal{G}:=\left(G_{0}, \Delta,\left\{\theta_{t}\right\}_{t \in \Delta}\right)$ of $G_{0}$ over $\Delta$ is projected to a holomorphic family $\hat{\mathcal{G}}$ of $G_{0}$ over the punctured disk $\Delta^{*}=\Delta /\langle\gamma\rangle=\{0<|z|<1\}$.

The holomorphic family $\hat{\mathcal{G}}$ of $G_{0}$ over $\Delta^{*}$ cannot be extended to a holomorphic family of $G_{0}$ over $\Delta$. Indeed, if it can be extended to a holomorphic family $\hat{\mathcal{G}}_{0}$ of $G_{0}$ over $\Delta$, then it consists of quasiconformal deformations of $G_{0}$. However, if we take $x \in(0,1)$ so that $x \rightarrow 0, \theta_{x}\left(G_{0}\right)$ gives a squeezing deformation of $X_{\Omega}$ about $\beta$. Thus, the hyperbolic lengths of the geodesics homotopic to $\beta$ tends to zero as $x \rightarrow 0$ (see [19]). Thus, the maximal dilatations of quasiconformal mappings which give isomorphisms from $G_{0}$ to $\theta_{x}\left(G_{0}\right)(0<x<1 / 2)$ are unbounded. It is a contradiction.

\section{The HOLOMORPHIC CONVEXITY OF DEFORMATION SPACES OF KLEINIAN GROUPS}

In this section, we shall prove Theorem III]

Let $G_{0}$ be a finitely generated Kleinian group. We give a proof of the first statement of the theorem. We show that in $D\left(G_{0}\right)$, the Carathéodory pseudo distance $c_{D\left(G_{0}\right)}$ is a distance. Namely, we show that $c_{D\left(G_{0}\right)}(x, y)>0$ if $x \neq y$.

For each $\gamma \in G_{0}$, we consider a function $f_{\gamma}(z)=\left(\operatorname{Tr} \rho_{z}(\gamma)\right)^{2}$, where $(\operatorname{Tr}(\alpha))^{2}$ stands for the square of the trace of $\alpha \in \operatorname{PSL}(2, \mathbb{C})$ and $\rho_{z}$ is the isomorphism given by $z \in D\left(G_{0}\right)$. The function $f_{\gamma}$ is holomorphic on $D\left(G_{0}\right)$ and if $\gamma$ is loxodromic, then the range is in $\mathbb{C} \backslash[0,4]$ because the isomorphisms $\rho_{z}$ are type preserving for $z \in D\left(G_{0}\right)$. Thus, $F_{\gamma}:=\varphi \circ f_{\gamma}$ is a bounded holomorphic function for a conformal map $\varphi$ from $\mathbb{C} \backslash[0,4]$ into $\Delta$.

Take any two distinct points $x, y$ in $D\left(G_{0}\right)$. Then the two points $x$ and $y$ give two different isomorphisms $\rho_{x}$ and $\rho_{y}$ of $\operatorname{Hom}\left(G_{0}, \operatorname{PSL}(2, \mathbb{C})\right)$, respectively. Moreover, the isomorphisms $\rho_{x}, \rho_{y}$ are not conjugate to each other since they are distinct points in $D\left(G_{0}\right)$. Hence, we may find a loxodromic element $\gamma \in G_{0}$ such that $F_{\gamma}(x) \neq F_{\gamma}(y)$ and we verify that $c_{D\left(G_{0}\right)}(x, y)>0$. So, the first statement of the theorem is proved.

Next, we show that the Carathédory distance on $D\left(G_{0}\right)$ is complete if every component of $G_{0}$ is simply connected.

Let $X_{0}$ be a hyperbolic Riemann surface of type $(g, n)$. Bers showed that the Teichmüller space $T\left(X_{0}\right)$ of $X_{0}$ is biholomorphically equivalent to a bounded domain in $\mathbb{C}^{3 g-3+n}$ (cf. [17, [11]). Since the Carathéodory distance in $T\left(X_{0}\right)$ is complete (cf. [3], [26]), the Teichmüller space $T\left(X_{0}\right)$ is $H^{\infty}$-convex. In fact, it is polynomial convex $([26])$.

Suppose that every component of $G_{0}$ is simply connected. It follows from Ahlfors' finiteness theorem(Theorem 2.2) that there are finitely many non-equivalent components, say $\Omega_{1}, \ldots, \Omega_{n}$, of $G_{0}$ and $X_{j}:=\Omega_{j} / G_{0, j}$ is a Riemann surface of finite type, where $G_{0, j}$ is a stabilized subgroup of $\Omega_{j}(j=1, \ldots, n)$. Since every $\Omega_{j}$ is simply connected, $D\left(G_{0}\right)$ is biholomorphically equivalent to the product of Teichmüller spaces $T\left(X_{1}\right) \times \ldots \times T\left(X_{n}\right)$, from a theorem of Kra and Maskit ([16]). 
Now, we consider the Carathéodory distance. Generally, in the product manifold $M=M_{1} \times \ldots \times M_{n}$ of complex manifolds $M_{1}, \ldots, M_{n}$, the Carathéodory distance $c_{M}$ satisfies

$$
c_{M}(a, b) \geq \max _{j=1, \ldots, n}\left\{c_{M_{j}}\left(a_{j}, b_{j}\right)\right\}
$$

for $a=\left(a_{1}, \ldots, a_{n}\right), b=\left(b_{1}, \ldots, b_{n}\right) \in M$. Actually, it is a direct consequence of the decreasing property of the Carathéodory pseudo distance (cf. [13]). Therefore, if the Carathéodory distances $c_{M_{j}}$ are complete, so is the Carathéodory distance $c_{M}$ and $M$ is $H^{\infty}$-convex.

Applying the above arguments for $D\left(G_{0}\right)$ which is biholomorphically equivalent to the product of Teichmüller spaces $T\left(X_{1}\right), \ldots, T\left(X_{n}\right)$, we conclude that the Carathéodory distance $c_{D\left(G_{0}\right)}$ is complete and we obtain the second statement of the theorem.

Finally, we show that the last statement of the theorem, that is, $D\left(G_{0}\right)$ is not $H^{\infty}$-convex and the Carathéodory distance is not complete if $G_{0}$ has a non-simply connected component.

Suppose that $G_{0}$ has a non-simply connected component. Then, from Theorem II we may construct a holomorphic family of $G_{0}$ over the punctured disk $\Delta^{*}$ but the holomorphic family cannot be extended to a holomorphic family of $G_{0}$ over $\Delta$. In fact, we construct a holomorphic map $F: \Delta^{*} \rightarrow D\left(G_{0}\right)$ which cannot be extended to a holomorphic map on $\Delta$.

Put $K=F(\{|t|=1 / 2\})$. Then $K$ is a compact subset of $D\left(G_{0}\right)$. For every bounded holomorphic function $f$ on $D\left(G_{0}\right), f \circ F$ is a bounded holomorphic function on $\Delta^{*}$. Therefore, it can be extended to a holomorphic function on $\Delta$. From the maximum principle, we have

$$
|f \circ F(t)| \leq \max _{|t|=1 / 2}|f \circ F(t)|
$$

for all $t$ with $0<|t| \leq 1 / 2$. Hence, $F(\{0<|t| \leq 1 / 2\})$ is contained in $H^{\infty}$ convex hull of $K$. Since $F$ cannot be extended to a holomorphic map on $\Delta$, $F(\{0<|t| \leq 1 / 2\})$ is not a compact subset of $D\left(G_{0}\right)$. Therefore, $D\left(G_{0}\right)$ is not $H^{\infty}$-convex and the Carathéodory distance is not complete.

Remark 6.1. As for the complex structure of $D\left(G_{0}\right)$, Kra and Maskit 16 showed that $D\left(G_{0}\right)$ is biholomorphically equivalent to a domain in $\mathbb{C}^{n}$ and the domain is holomorphically convex. We have seen in Theorem III that if every component of $G_{0}$ is simply connected, then $D\left(G_{0}\right)$ is $H^{\infty}$-convex, which is a stronger property than the holomorphic convexity. On the other hand, we show that the situation is completely different when $G_{0}$ has a non-simply connected component.

\section{Proof of Theorem IV]}

We show that the Teichmüller distance $d_{T}^{D\left(G_{0}\right)}$ of $D\left(G_{0}\right)$ coincides with the Kobayashi distance as the Teichmüller distance $d_{T}$ of the Teichmüller space. The proof is done by using the same argument as in Earle-Kra-Krushkal' (4]) for Teichmüller spaces.

Let $\mathcal{M}\left(G_{0}\right)$ be the space of $G_{0}$-compatible Beltrami coefficients on $\hat{\mathbb{C}}$. For $\rho_{1}, \rho_{2} \in D\left(G_{0}\right)$, we may take an extremal quasiconformal map $w_{1,2}$ for the Teichmüller distance. Thus, we have

$$
d_{T}^{D(g)}\left(\rho_{1}, \rho_{2}\right)=\log K\left(w_{1,2}\right)=\log \frac{1+\left\|\mu_{1,2}\right\|_{\infty}}{1-\left\|\mu_{1,2}\right\|_{\infty}},
$$


where $\mu_{1,2}$ is the Beltrami coefficient of $w_{1,2}$. We consider a holomorphic map $\Phi: \Delta \rightarrow D\left(G_{0}\right)$ defined by

$$
\Phi(t)=\left[t \frac{\mu_{1,2}}{\left\|\mu_{1,2}\right\|}\right]
$$

where $[\mu]\left(\mu \in \mathcal{M}\left(G_{0}\right)\right)$ is an element of $D\left(G_{0}\right)$ given by a quasiconformal map whose Beltrami coefficient is $\mu$. Then, from the definition of the Kobayashi distance, we have

$$
d_{K}^{D(g)}\left(\rho_{1}, \rho_{2}\right) \leq \rho_{\Delta}\left(0,\|\mu\|_{\infty}\right)=d_{T}^{D\left(G_{0}\right)}\left(\rho_{1}, \rho_{2}\right) .
$$

For every holomorphic map $f: \Delta \rightarrow D(G)$ with $f(0)=\rho_{1}$ and $f\left(t_{0}\right)=\rho_{2}$, we have a holomorphic map $g: \Delta \rightarrow \mathcal{M}\left(G_{0}\right)$ such that

$$
f=\Phi \circ g \text {. }
$$

It is a consequence of Theorem 3.2. Hence, we have

$$
\rho_{\Delta}\left(0, t_{0}\right) \geq d_{\mathcal{M}\left(G_{0}\right)}\left(g(0), g\left(t_{0}\right)\right),
$$

where $d_{\mathcal{M}\left(G_{0}\right)}$ is the Kobayashi distance of $\mathcal{M}\left(G_{0}\right)$. On the other hand, it is shown that it is the same as the Teichmüller distance of $\mathcal{M}\left(G_{0}\right)$ by using the same argument as in 4 Proposition 1. Therefore, by taking the infimum of the left-hand side over all holomorphic maps $f$, we have the opposite inequality of (7.1). This completes the proof of the theorem.

Remark 7.1. As we have stated in the previous section, the deformation space $D\left(G_{0}\right)$ is biholomorphically equivalent to a product of Teichmüller spaces if every component is simply connected. In this case, the coincidence of the two distances is known. In fact, they are the same distance on product Teichmüller spaces with an arbitrary index set ([7]). Indeed, the method in the proof of Proposition 7.28 in 7. also shows that the the infinitesimal Teichmülller metric and the infinitesimal Kobayashi metric are equal on $D\left(G_{0}\right)$, which was suggested by the referee.

\section{ACKNOWLEDGEMENT}

The author thanks the referee for the careful reading of the manuscript and valuable suggestions that helped to improve the paper.

\section{REFERENCES}

[1] Michael Beck, Yunping Jiang, Sudeb Mitra, and Hiroshige Shiga, Extending holomorphic motions and monodromy, Ann. Acad. Sci. Fenn. Math. 37 (2012), no. 1, 53-67, DOI 10.5186/aasfm.2012.3713. MR2920423

[2] Lipman Bers, Holomorphic families of isomorphisms of Möbius groups, J. Math. Kyoto Univ. 26 (1986), no. 1, 73-76. MR827159 (87j:32067)

[3] Clifford J. Earle, On the Carathéodory metric in Teichmüller spaces, Discontinuous groups and Riemann surfaces (Proc. Conf., Univ. Maryland, College Park, Md., 1973), Princeton Univ. Press, Princeton, N.J., 1974, pp. 99-103. Ann. of Math. Studies, No. 79. MR.0352450 (50 \#4937)

[4] C. J. Earle, I. Kra, and S. L. Krushkal', Holomorphic motions and Teichmüller spaces, Trans. Amer. Math. Soc. 343 (1994), no. 2, 927-948, DOI 10.2307/2154750. MR.1214783(94h:32035)

[5] Clifford J. Earle and Albert Marden, On holomorphic families of Riemann surfaces, Conformal dynamics and hyperbolic geometry, Contemp. Math., vol. 573, Amer. Math. Soc., Providence, RI, 2012, pp. 67-97, DOI 10.1090/conm/573/11413. MR2964074

[6] Clifford J. Earle and Curt McMullen, Quasiconformal isotopies, Holomorphic functions and moduli, Vol. I (Berkeley, CA, 1986), Math. Sci. Res. Inst. Publ., vol. 10, Springer, New York, 1988, pp. 143-154, DOI 10.1007/978-1-4613-9602-4_12. MR955816 (89h:30028) 
[7] Clifford J. Earle and Sudeb Mitra, Variation of moduli under holomorphic motions, In the tradition of Ahlfors and Bers (Stony Brook, NY, 1998), Contemp. Math., vol. 256, Amer. Math. Soc., Providence, RI, 2000, pp. 39-67, DOI 10.1090/conm/256/03996. MR1759669 (2001f:30031)

[8] Robert C. Gunning, Introduction to holomorphic functions of several variables. Vol. I, The Wadsworth \& Brooks/Cole Mathematics Series, Wadsworth \& Brooks/Cole Advanced Books \& Software, Pacific Grove, CA, 1990. Function theory. MR1052649 (92b:32001a)

[9] John Hamal Hubbard, Sur les sections analytiques de la courbe universelle de Teichmüller, Mem. Amer. Math. Soc. 4 (1976), no. 166, ix+137. MR0430321 (55 \#3326)

[10] John Hamal Hubbard, Teichmüller theory and applications to geometry, topology, and dynamics. Vol. 1, Matrix Editions, Ithaca, NY, 2006. Teichmüller theory; With contributions by Adrien Douady, William Dunbar, Roland Roeder, Sylvain Bonnot, David Brown, Allen Hatcher, Chris Hruska and Sudeb Mitra; With forewords by William Thurston and Clifford Earle. MR 2245223 (2008k:30055)

[11] Y. Imayoshi and M. Taniguchi, An introduction to Teichmüller spaces, Springer-Verlag, Tokyo, 1992. Translated and revised from the Japanese by the authors. MR,1215481 (94b:32031)

[12] Yunping Jiang and Sudeb Mitra, Some applications of universal holomorphic motions, Kodai Math. J. 30 (2007), no. 1, 85-96, DOI 10.2996/kmj/1175287624. MR2319079 (2008c:32019)

[13] Shoshichi Kobayashi, Hyperbolic manifolds and holomorphic mappings, Pure and Applied Mathematics, vol. 2, Marcel Dekker, Inc., New York, 1970. MR0277770 (43 \#3503)

[14] Irwin Kra, On spaces of Kleinian groups, Comment. Math. Helv. 47 (1972), 53-69. MR0306485 (46 \#5611)

[15] Irwin Kra, Deformation spaces, A crash course on Kleinian groups (Lectures at a Special Session, Annual Winter Meeting, Amer. Math. Soc., San Francisco, Calif., 1974), Springer, Berlin, 1974, pp. 48-70. Lecture Notes in Math., Vol. 400. MR0402122 (53 \#5943)

[16] Irwin Kra and Bernard Maskit, The deformation space of a Kleinian group, Amer. J. Math. 103 (1981), no. 5, 1065-1102, DOI 10.2307/2374258. MR630778 (83e:32026)

[17] Olli Lehto, Univalent functions and Teichmüller spaces, Graduate Texts in Mathematics, vol. 109, Springer-Verlag, New York, 1987. MR867407 (88f:30073)

[18] R. Mañé, P. Sad, and D. Sullivan, On the dynamics of rational maps, Ann. Sci. École Norm. Sup. (4) 16 (1983), no. 2, 193-217. MR732343 (85j:58089)

[19] Albert Marden and Howard Masur, A foliation of Teichmüller space by twist invariant disks, Math. Scand. 36 (1975), no. 2, 211-228. MR0393584 (52 \#14393)

[20] Bernard Maskit, A theorem on planar covering surfaces with applications to 3-manifolds, Ann. of Math. (2) 81 (1965), 341-355. MR0172252 (30 \#2472)

[21] Bernard Maskit, Self-maps on Kleinian groups, Amer. J. Math. 93 (1971), 840-856. MR.0291453 (45 \#544)

[22] Katsuhiko Matsuzaki and Masahiko Taniguchi, Hyperbolic manifolds and Kleinian groups, Oxford Mathematical Monographs, The Clarendon Press, Oxford University Press, New York, 1998. Oxford Science Publications. MR 1638795 (99g:30055)

[23] Curtis T. McMullen, Complex earthquakes and Teichmüller theory, J. Amer. Math. Soc. 11 (1998), no. 2, 283-320, DOI 10.1090/S0894-0347-98-00259-8. MR1478844 (98i:32030)

[24] Sudeb Mitra and Hiroshige Shiga, Extensions of holomorphic motions and holomorphic families of Möbius groups, Osaka J. Math. 47 (2010), no. 4, 1167-1187. MR2791561|(2012f:37099)

[25] Robert Riley, Holomorphically parameterized families of subgroups of SL(2, C), Mathematika 32 (1985), no. 2, 248-264 (1986), DOI 10.1112/S0025579300011037. MR834494 (87f:32056)

[26] Hiroshige Shiga, On analytic and geometric properties of Teichmüller spaces, J. Math. Kyoto Univ. 24 (1984), no. 3, 441-452. MR766636 (86c:32024)

[27] Zbigniew Slodkowski, Holomorphic motions and polynomial hulls, Proc. Amer. Math. Soc. 111 (1991), no. 2, 347-355, DOI 10.2307/2048323. MR.1037218 (91f:58078)

[28] Dennis Sullivan, Quasiconformal homeomorphisms and dynamics. II. Structural stability implies hyperbolicity for Kleinian groups, Acta Math. 155 (1985), no. 3-4, 243-260, DOI 10.1007/BF02392543. MR806415 (87i:58104)

Department of Mathematics, Tokyo Institute of Technology, O-okayama, MeguroKU TOKYO, JAPAN

E-mail address: shiga@math.titech.ac.jp 